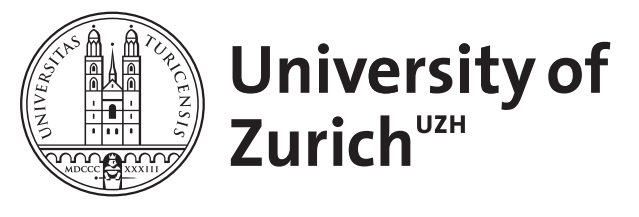

\title{
Bildgebung beim Schlaganfall
}

Winklhofer, Sebastian F X ; Kulcsár, Zsolt

\begin{abstract}
In recent decades, imaging has become indispensable for the diagnosis and therapy of stroke. It provides information necessary to distinguish between hemorrhagic and ischemic stroke, to identify the cause of the symptoms and to determine the time of stroke. Increasingly, imaging is being used as a tool for making optimal therapeutic decisions. In particular, the advent and success of mechanical thrombectomy has significantly changed the management of patients with ischemic stroke. Computed tomography (CT) and magnetic resonance imaging (MRI) allow for identifying patients who may benefit from revascularizing therapy, even outside the previously valid, dogmatic time windows. Advances in imaging modalities both in CT and MRI, allow for identifying salvageable brain tissue, independent from the time factor, advocating individualized decision making for optimal therapy and prognosis. The choice of the appropriate imaging modality depends on several criteria, such as suspected and differential diagnosis, urgency, patient age and availability of the scanner.
\end{abstract}

DOI: https://doi.org/10.1024/0040-5930/a001150

Other titles: Stroke Imaging

Posted at the Zurich Open Repository and Archive, University of Zurich ZORA URL: https://doi.org/10.5167/uzh-188923

Journal Article

Accepted Version

Originally published at:

Winklhofer, Sebastian F X; Kulcsár, Zsolt (2020). Bildgebung beim Schlaganfall. Therapeutische Umschau. Revue thérapeutique, 77(2):39-46.

DOI: https://doi.org/10.1024/0040-5930/a001150 
Klinik für Neuroradiologie, UniversitätsSpital Zürich, Zürich

\section{Bildgebung bei Schlaganfall}

PD Dr. Sebastian F.-X. Winklhofer

PD Dr. Zsolt Kulcsár

Klinik für Neuroradiologie

Klinisches Neurozentrum

UniversitätsSpital Zürich

Frauenklinikstrasse 10

$\mathrm{CH}-8091$ Zürich

sebastian.winklhofer@usz.ch

Telefon: 044-255 1111 


\section{Zusammenfassung}

Die neuroradiologische Bildgebung ist in den letzten Jahrzehnten für die Diagnose und Therapie des Schlaganfalls unverzichtbar geworden. Sie liefert die notwendigen Informationen, um zwischen hämorrhagischem und ischämischem Schlaganfall zu unterscheiden, die Ursache der Symptome zu identifizieren und den Zeitpunkt des Schlaganfalls zu bestimmen. Zunehmend ist die Bildgebung hilfreich, um therapeutische Entscheidungen optimal treffen zu können. Insbesondere das Aufkommen und die erfolgreiche Etablierung der mechanischen Thrombektomie hat die Behandlung von Patienten mit ischämischem Schlaganfall erheblich verändert. Mit Hilfe der Computertomographie (CT) und der Magnetresonanztomographie (MRT) können Patienten identifiziert werden, die von einer revaskularisierenden Therapie profitieren können, auch ausserhalb des bisher gültigen, dogmatischen Zeitfensters. Fortschritte bei den bildgebenden Verfahren sowohl in der CT als auch in der MRT ermöglichen die Identifizierung von rettbarem Hirngewebe, unabhängig vom Zeitfaktor und begünstigen eine individualisierte Entscheidungsfindung für eine optimale Therapie und Prognose. Die Wahl der geeigneten Bildgebungsmodalität hängt von mehreren Kriterien ab, wie z.B. Verdachts- und Differentialdiagnose, Dringlichkeit, Patientenalter und Verfügbarkeit des Scanners.

\section{Summary}

In recent decades, imaging has become indispensable for the diagnosis and therapy of stroke. It provides information necessary to distinguish between hemorrhagic and ischemic stroke, to identify the cause of the symptoms and to determine the time of stroke. Increasingly, imaging is being used as a tool for making optimal therapeutic decisions. In particular, the advent and success of mechanical thrombectomy has significantly changed the management of patients with ischemic stroke. Computed tomography (CT) and magnetic resonance imaging (MRI) allow for identifying patients who may benefit from revascularizing therapy, even outside the previously valid, dogmatic time windows. Advances in imaging modalities both in CT and MRI, allow for identifying salvageable brain tissue, independent form the time factor, advocating individualized decision making for optimal therapy and prognosis. The choice of the appropriate imaging modality depends on several criteria, such as suspected and differential diagnosis, urgency, patient age and availability of the scanner. 


\section{Einführung}

Nach Angaben der Weltgesundheitsorganisation (WHO) ist der Schlaganfall weltweit die zweit-häufigste Todesursache und die dritt-häufigste Ursache für eine langfristige Behinderung (1).

In der Schweiz erleiden etwa 15'000 Patienten jährlich einen Schlaganfall und weitere 5'000 Patienten leiden unter einer transienten ischämischen Attacke (TIA) (2,3). Darüber hinaus ist der Schlaganfall nicht nur mit einer grossen Herausforderung für die betroffenen Patienten und deren Familien verbunden, sondern stellt auch eine grosse finanzielle Belastung für das Gesundheitssystem dar (4). In ca. 80\% der Schlaganfälle handelt es sich um einen ischämischen Infarkt, wohingegen ca. 20\% der Fälle hämorrhagisch bedingt sind.

Die Bildgebung des zentralen Nervensystems ist ein wesentlicher Bestandteil für die Beurteilung und Behandlung von Patienten, bei denen ein akuter hämorrhagischer oder ischämischer Schlaganfall vermutet wird. In den letzten Jahren verlagerte sich die Aufgabe der Bildgebung des ischämischen Schlaganfalls von der vormals dominierenden Intention, eine intrakranielle Blutung auszuschliessen, zu einem inzwischen weit fortgeschrittenen und umfassenderen Ansatz. Heutzutage ermöglichen moderne Konzepte der Schlaganfall-Bildgebung eine zeitnahe und präzise Diagnose, optimieren das Patientenmanagement, erweitern das mögliche individuelle Behandlungsspektrum und dienen der Prognosestellung $(5,6)$. Der Nutzen der verfügbaren Bildgebungsmodalitäten wie die Computertomographie (CT) oder die Magnetresonanztomographie (MRT) hat sich als unersetzlich erwiesen, diejenigen Patienten zu identifizieren, die möglicherweise von einem bestimmten Therapieansatz profitieren können. Jüngste klinische Studien zeigten gute klinische Ergebnisse der intravenösen (i.v.) thrombolytischen medikamentösen Therapie und vor allem der neurointerventionellen mechanischen Thrombektomie bei Patienten mit akutem Schlaganfall, die auf der Grundlage von Bildgebungskriterien ausgewählt wurden (7). So konnte mittels nicht-invasiver Gefäßdarstellung und Perfusionsbildgebung auch das zeitliche Therapiefenster in den vergangenen Jahren deutlich erweitert werden und damit Patienten eine Therapie zukommen lassen, die ihnen vormals verwehrt gewesen wäre $(8,9)$.

Im Folgenden soll zunächst auf die zur Verfügung stehenden Bildmodalitäten und anschliessend auf deren Verwendung beim hämorrhagischen und ischämischen Schlaganfall eingegangen werden.

\section{Bildgebungsmodalitäten}




\section{Wann CT und wann MRT?}

Die Frage welche Modalität besser zur Bildgebung des Schlaganfalls geeignet ist wird rege diskutiert. Die Hauptaufgabe der neuroradiologischen Abklärung beim akuten Schlaganfall setzt sich aus folgenden drei Punkten zusammen: 1. Diagnosestellung und damit Festlegung, ob hämorrhagischer oder ischämischer Schlaganfall sowie Ausschluss sonstiger Differentialdiagnosen. 2. Identifikation von Patienten, die von einer bestimmten Behandlung profitieren können (Revaskularisation möglich? Blutungsquelle und erneutes Blutungsrisiko?). 3. Hilfe bei der Wahl der richtigen Behandlung (i.v. Lyse, mechanische Thrombektomie beim ischämischen Schlaganfall, chirurgische oder endovaskuläre Behandlung einer hämorrhagischen Gefässanomalie).

Insbesondere beim akuten ischämischen Schlaganfall liegt aufgrund des rapiden Untergangs der Neuronen ein Hauptaugenmerk auf der Schnelligkeit. Das Motto «Time is Brain» gilt auch heute weiter noch. Damit und in Zusammenschau mit den oben genannten Punkten sind folgende Voraussetzungen an die Bildgebung zu stellen: 1. Verfügbarkeit und Zugänglichkeit rund um die Uhr (24/7). 2. Schnelligkeit. 3. Validität um Fragestellungen beantworten zur können. 4. Einfache Interpretation ohne lange Nachbearbeitung.

Grundsätzlich erlauben beide etablierte Modalitäten den oben genannten Anforderungen gerecht zu werden. Auch die aktuelle Studienlage zeigt keinen klaren Gewinner, wenn es um die Frage der besseren Modalität geht $(10,11)$. Das verwendete Verfahren hängt im Wesentlichen von den vor Ort gegebenen Verfügbarkeiten und dem Kosten-Nutzen Verhältnis ab.

\section{Computertomographie}

CT ist nach wie vor das meist verwendete Schnittbildverfahren bei der Bildgebung des akuten Schlaganfalls. Vorteile sind die gute Verfügbarkeit, die schnelle Durchführung der Untersuchung (wenige Minuten), hoch-aufgelöste Darstellung der supra-aortalen und intrakraniellen Gefässen und die geringeren Kosten im Vergleich zur MRI Bildgebung. Nachteile sind die im Vergleich zur MRT eingeschränkte Sensitivität für Läsionen des Hirnparenchyms, die meist notwendige Gabe von Kontrastmittel (KM) sowie die einhergehende Strahlenbelastung. Zu den Kontraindikationen für ein CT zählt im Allgemeinen eine Schwangerschaft, wobei im individuellen, akuten Fall und bei strenger Indikation eine CT Untersuchung unausweichlich sein kann. Bei notwendiger Kontrastmittelgabe muss an eine mögliche Unverträglichkeit oder Allergie, Niereninsuffizienz und an pathologische Schilddrüsenwerte gedacht werden. 
Die zur Verfügung stehenden Optionen beinhalten das Nativ-CT des Neurokraniums, die arterielle und venöse intra- und der extrakranielle Gefässdarstellung mittels jodhaltigem KM (CT Angiographie, CT-A), das Schädel CT in venöser (Spät-)Phase sowie die KMgestützte CT Perfusion (CT-P).

\section{Magnetresonanztomographie}

MRT als allgemein initiale Bildgebungsmodalität beim akuten Schlaganfall findet zum aktuellen Zeitpunkt in relativ wenigen Institutionen statt. Nur wenige Einrichtungen haben die dafür notwendige Infrastruktur (Verfügbarkeit 24/7 inkl. notwendigem Personal). Falls beide Modalitäten verfügbar sind (wie bei den meisten Stroke Centern in der Schweiz der Fall), kann bei Bedarf zwischen den Modalitäten ausgewählt werden, um die jeweiligen Vorteile bestmöglich auszunützen. Dennoch spielt die MR Bildgebung bereits eine zentrale Rolle in der Schlaganfallabklärung. Hauptvorteil ist die Möglichkeit der detaillierten Darstellung der Gehirnparenchyms mittels der unterschiedlichen zur Verfügung stehenden Sequenzen sowie die für den Patienten strahlenfreie Bildakquisition. Hauptnachteile sind die längere Untersuchungsdauer und die ggf. limitierte Verfügbarkeit, was insbesondere bei der akuten Schlaganfalldiagnostik berücksichtigt werden muss.

Absolute Kontraindikationen für eine MR-Untersuchung sind heutzutage vor allem bei richtiger Indikationsstellung nicht mehr pauschal aufzulisten. Viele der heute verwendeten Implantate (z.B. Herzschrittmacher) sind unter Berücksichtigung der Herstellerangaben und ggf. entsprechender Überwachung des Patienten MR-gängig. Zu den relativen Kontraindikationen zählen eine Schwangerschaft (erstes Trimenon), grosse Tätowierungen sowie Klaustrophobie. Bei KM Gabe (Gadolinium) ist bei schwerer Niereninsuffizienz oder Dialysepflicht an die Nephrogene Systemische Fibrose zu denken, wenngleich diese Nebenwirkung heutzutage nur noch selten auftritt (14). Je nach Indikation und Fragestellung erlaubt das MRT neben den klassischen T1 und T2 gewichteten Sequenzen mit seiner Vielzahl an weiteren Sequenzen Struktur und Funktion aus unterschiedlichen physikalischen Perspektiven zu betrachten. Hierzu zählen im klinischen Alltag vor allem die folgenden Akquisitionen: DWI (diffusion weighted imaging), FLAIR (fluid attenuation inversion recovery), SWI (susceptibilityweighted imaging), TOF (time-of-flight) MR-Angiographie und die MR-Perfusion (MR-P). MR Protokolle werden der jeweiligen Situation und den Gegebenheiten vor Ort angepasst. Schnelle Protokolle (d.h. z.B. zur akuten Schlaganfallabklärung in unter 10 min. Scanzeit) bestehen aus DWI, FLAIR und SWI Sequenzen mit optionaler TOF Gefässdarstellung und MR-P. Insbesondere kann ein kurzes Protokoll ergänzend zum CT durchgeführt werden, falls dort Unklarheiten bestehen. Protokolle zur Abklärung bei 
subakuten oder chronischen Infarkten und deren Differentialdiagnosen (z.B. stroke mimiker wie Vaskulitis oder Vasospasmen) enthalten zusätzlich T1w (nativ und mit KM) und T2w Sequenzen.

Im Falle der Verfügbarkeit von CT und MRT kann beim akuten Schlaganfall die Modalität auf den individuellen Fall, also mit Hinblick auf die Symptomatik des Patienten gewählt werden. Allgemeingültige Aussagen sind schwierig zu tätigen, allerdings gibt es Gegebenheiten, die eine jeweilige Modalität bevorzugen: z.B. MRT bei Wake-up Stroke Abklärung, subakuter oder chronischer Schlaganfall, Kinder oder junge Patienten, Schwangerschaft, vermutete Ischämie in der hinteren Gefässzirkulation (hier ist die MRT der CT überlegen (12)) (Tab. 1).

Im Folgenden soll auf den wesentlichen Nutzen dieser unterschiedlichen Akquisitionen für die neuroradiologische Abklärung eines Schlaganfalls näher eingegangen werden.

\section{Bildgebung des hämorrhagischen Schlaganfalls}

\section{Nativ CT}

Das nativ CT des Schädels ist trotz seiner Einfachheit immer noch unverzichtbar. Insbesondere Stammganglienblutungen und Lobärblutungen sind klinisch oft nicht vom klassischen Erscheinungsbild eines ischämischen Infarktes zu unterscheiden. Die Kernfrage, ob es sich um einen hämorrhagischen oder einen ischämischen Schlaganfall handelt kann mittels nativ CT schnell, einfach und zuverlässig beantwortet werden. Eine akute intrakranielle Blutung stellt sich hyperdens (= hell) im CT dar. Je nach Alter der Blutung kann diese auch iso- oder hypodens erscheinen (z.B. chronisches Subduralhämatom). Abb. 1 zeigt Beispiele für die häufigsten Blutungsformen im CT. Die Bildgebung erlaubt die Darstellung des Ausmasses der Blutung, eine Altersabschätzung (Dichteabnahme im Verlauf in Hounsfield Einheiten gemessen) und ggf. den Nachweis einer zugrunde liegenden Pathologie. Auch andere Befunde können im CT sichtbar sein, z.B. erweiterte Liquorräume (Hydrocephalus), vasogenes Ödem, Leukenzaphalopathie, ältere lakunäre (=unter $1 \mathrm{~cm}$ Durchmesser) oder territoriale Infarkte.

\section{CT-Angiographie und venöse Phase}

CT gilt als häufigste Modalität der Wahl bei der initialen Abklärung bei Blutungsverdacht. Bei neu detektierter Hirnblutung sollte eine Darstellung der Gefässe zum Ausschluss einer zugrundeliegenden Gefässpathologie (z.B. Aneurysma, durale arteriovenöse Fistel, arteriovenöse Malformation) durchgeführt werden. Mittels KM unterstützter CT in venöser Phase lassen sich Thrombosen der venösen Blutleiter oder Venen nachweisen. 
Zeigt sich ein KM-Austritt innerhalb einer Parenchymblutung, so ist dies möglicherweise Zeichen einer aktiven Blutung (Spot Sign). Ausserdem lassen sich potentiell zugrundeliegende eingeblutete Neoplasien darstellen, wie es z.B. häufig bei Melanommetastasen beobachtet wird.

\section{$M R T$}

MRT bietet mit seiner Vielfalt an optionalen Sequenzen zusätzliche Vorteile, wie z.B. bei der Suche von oben erwähnten potentiellen Blutungsursachen. Kleine Metastasen, Gefässmalformationen oder andere Pathologien können meist besser als im CT dargestellt werden, so dass ein MRT bei unklarer Blutungsursache im Verlauf ergänzt werden sollte. Auch lässt sich mittels MRT das Blutungsalter besser als im CT abschätzen, wenngleich sich dies, aufgrund der unterschiedlichen Sequenzen und des unterschiedlichen Signalverhaltens der verschiedenen Blutabbauprodukte, als nicht immer einfach erweist. Z.B. zeigt sich intrazelluläres Deoxyhämoglobin einer akuten Blutung in der T1w Sequenz von intermediärem Signal, wobei es in der T2w Sequenz eher hypointens zur Darstellung kommt (15). Mittels SWI Sequenzen lassen sich neben grösseren Einblutungen vor allem kleinste Mikroblutungen nachweisen, die bei zentraler Häufung eher für eine hypertensive Ätiologie sprechen, wohingegen bei peripherer Häufung eher eine amyloid assoziierte Blutung in Anbetracht kommt (zerebrale Amyloidangiopathie).

\section{Bildgebung des ischämischen Schlaganfalls}

Computertomographie

\section{Nativ-CT}

Ist im Akutfall eine Blutung im nativ CT ausgeschlossen, so ist auf Zeichen eines cerebrovaskulären ischämischen Insults zu achten. Hier ist das Parenchym nach den klassischen Ischämie Frühzeichen zu untersuchen: Verstrichenes Mark-Rinden Band, hyperdenses Arterien Zeichen, verminderte Differenzierbarkeit der Grauen- und Weissen Substanz, Abgrenzung von flauen Hypodensitäten und verminderte Abgrenzbarkeit der Basalganglien (Abb. 2). Eine hyperakute Ischämie ist jedoch nicht immer im nativ CT nachweisbar. Insbesondere der Nachweis von ischämischem Parenchym kann in den ersten Stunden nach Symptombeginn negativ ausfallen. Der Nachweis hängt hier, neben der Zeit, auch vom Ausmass des betroffenen Hirnparenchyms, kollateraler Blutversorgung und von der Erfahrenheit des Untersuchers ab. Das zytotoxische Ödem eines ischämischen Infarktes stellt sich als flaue 
Hypodensität dar und lässt sich als erstes meist bei Befall der tiefen Grauen Substanz (Basalganglien) nachweisen (ab einer Stunde nach Gefässverschluss in 60\% der Fälle aufgrund der fehlenden Kollateralversorgung). Grössere infarzierte Areale wie z.B. bei einem Verschluss der A. cerebri media lassen sich in der Regel nach 4-6 Stunden gut abgrenzen (16, 17). Neben dem (hyper-) akuten Schlaganfall sind in zeitlicher Abhängigkeit unterschiedliche Infarktstadien zu differenzieren (Tab. 2, Abb. 2b, 2c) (15). Diese Unterscheidung ist vor allem zur Auswahl der geeigneten Therapie entscheidend. Bereits eindeutig hypodens demarkiertes Hirnparenchym entspricht meist irreversibel geschädigtem Gewebe (zytotoxisches Ödem). Auch der Nachweis von mehrzeitigen Ereignissen, wie z.B. stummen Infarkten kann klinisch von Bedeutung sein und ist im CT möglich.

\section{CT-Angiographie}

Die detaillierte Darstellung der supra-aortalen und zerebralen Gefässen und deren möglicher Pathologien wie Stenosen, Dissektionen und Okklusionen, ist der grossen Vorteil des CT. Mittels CT-A der intra- und extrakraniellen Gefässe nach i.v. KM Gabe soll vor allem die potentielle Ätiologie eines ischämischen Infarktes aufgezeigt werden. Dazu zählen insbesondere thrombotische Gefässverschlüsse bei arteriosklerotischen Gefässwandveränderungen, kardiale Embolien oder Thrombosen der Hirnvenen oder der grossen venösen Blutleiter (z.B. Sinus transversus). Ein thrombo-embolischer Verschluss zeigt sich in einem KM-Abbruch des Gefässes wie z.B. der A. cerebri media. Die genaue Lokalisation hiervon ist auch in Anbetracht einer möglichen mechanischen Thrombektomie wichtig (z.B. durch Angabe des betroffenen Gefässsegments der A. cerebri media wie M1-M4). Stenosen sind vor allem auch an den extrakraniellen Gefässen häufig und als mögliche Ursache der Beschwerden zu orten. Prädilektionsstelle ist der Bulbus der A. carotis interna an der Karotisbifurkation. In diesem Zusammenhang ist als weitere häufige Schlaganfallursache eine Dissektion der grossen Gefässe auszuschliessen. Diese treten häufiger extrakraniell unter der Schädelbasis oder in der A. vertebralis entlang der Atlasschleife bzw. im Verlauf innerhalb der Foramina transversaria (18) auf. Bildmorphologisch können dissezierte Gefässe mit einem Verschluss, einer Stenose, Irregularitäten der Gefässwand und einem Wandhämatom einhergehen, jeweils mit oder ohne cerebrale Embolien.

Je nach Akquisitionsphase kann der als zunehmend wichtig eingestufte Status der Kollateralgefässe analysiert werden: kräftig ausgeprägte Kollateralen über die Gegenseite oder ein schneller retrograder Blutfluss durch leptomeningeale Kollateralen von anderen Versorgungsgebieten sind als gute prognostische Faktoren anzusehen. 


\section{$C T$ - venöse Kontrastmittelspätphase}

Die venöse Spätphase kann bei Bedarf zum Ausschluss anderer Ursachen der Symptomatik angefertigt werden. Dazu zählen z.B. die Sinus- oder Venenthrombosen oder intrakranielle Tumore. Nicht alle Differentialdiagnosen lassen sich mit der CT sicher nachweisen, so sind z.B. Encephalitiden oder Meningitiden nicht notwendigerweise im CT sichtbar.

\section{CT-Perfusion und Konzept der Penumbra}

Nach Durchführung der nativen CT und der CT-A erfolgt in Abhängigkeit der jeweiligen klinischen Situation die Akquisition einer CT-P. Diese gilt als funktionelle Aufnahme und hat sich inzwischen in der Bildgebung des akuten Schlaganfalls etabliert und ist in den entsprechenden Leitlinien je nach klinischer Situation fest verankert (19, 20). Mittels Perfusion wird die Durchblutung des Gehirns untersucht und minderversorgtes Parenchym nachgewiesen. Nach KM-Injektion und anschliessender repetitiver Bildakquisition über ca. 40-60 Sekunden wird das An- und Abfluten des KM gemessen, also die Passage des KM durch die Gefässe und das Gewebe. Es handelt sich um eine 4-D Bildgebung, die neben der 3-dimensionalen morphologischen Bildinformation zusätzlich auch den Zeitfaktor erfasst. Direkt anschliessend werden mittels Nachbearbeitung farbcodierte Parameterkarten erstellt, in denen die Kerngrössen der CT-P bildlich dargestellt werden. Moderne Scannersoftware erlaubt dies ohne wesentliche Zeitverzögerung um unnötige Wartezeit zu vermeiden. Die Kernparameter bestehen je nach verwendetem Rekonstruktionsalgorithmus aus dem cerebralen Blutfluss (CBF), dem cerebralen Blutvolumen (CBV), der mittleren Transitzeit (mean transit time, MTT), der Zeit-zum-Gipfel (time to peak, TTP) und der Zeit-zum-Maximum (time to maximum, Tmax) (Tab. 3).

Die zwei Hauptvorteile der CT-P sind die Detektion von frischen und teils auch kleinen Ischämien sowie Hilfestellung bei der Triage zur optimalen Therapie. Letzteres basiert auf der Möglichkeit gesundes, noch zu rettendes Hirngewebe, von bereits irreversibel infarziertem Gewebe zu differenzieren. Dieses Konzept hat die strikte Anwendung von Zeitfenstern zur Therapie annähernd abgelöst und ermöglicht nun eine bildgebungsbasierte patienten-individuelle optimale Therapie.

Farbkodierte MTT, TTP oder Tmax Karten der CT-P können im Rahmen der Bildinterpretation Areale einer zeitlich verzögerten Durchblutung darstellen. In der CBF und CBV Karte kann der irreversibel geschädigte Infarktkern identifiziert werden: nichtoder ungenügend mit Blut versorgtes Parenchym wird keine oder nur eine sehr langsam auftretende KM Anreicherung zeigen. Das den Infarktkern umgebende Gewebe, das womöglich ebenfalls mit Sauerstoff minderversorgte Nervenzellen beinhaltet, wird als 
Penumbra bezeichnet («tissue at risk»). Dieses Gewebe ist noch nicht irreversibel geschädigt und enthält noch intakte Nervenzellen, die via Restperfusion oder Kollateralen versorgt werde. Die Penumbra kann ebenfalls mittels der CT-P identifiziert werden. Vereinfacht gesagt, erfolgt dies aus der Differenz des betroffenen Hirnareals in MTT, TTP oder Tmax und dem irreversibel geschädigten Gewebe in der CBF/CBV. Die Differenz zwischen Infarktkern und Penumbra wird als Mismatch bezeichnet. Patienten ohne grösseres Mismatch in der Bildgebung von einer revaskularisierenden Therapie weniger gut profitieren als Patienten mit grossem Mismatch (Abb. 3-5). Dieses Konzept überwiegt klinisch den vormals üblichen Zeitfenstern, so dass nun auch Patienten bis zu 24 Stunden oder länger nach Symptombeginn von einer revaskularisierenden Therapie profitieren können.

\section{Magnetresonanztomographie}

\section{T1w und T2w}

T1w native Sequenzen dienen vor allem zum Nachweis von intrakraniellen Blutungen. Zudem sind sie für den Vergleich zur KM T1w Sequenz erforderlich, um damit eine tatsächliche KM-Anreicherung nachzuweisen. Durch die Gabe von KM können zudem pathologische Anreicherungen nachgewiesen werden. T2w Sequenzen sind besonders für eine initiale anatomische Orientierung und als Suchsequenz hilfreich. Auch kann mit ihr bereits ischämisch demarkiertes Hirngewebe oder altes Infarktgewebe identifiziert werden.

\section{FLAIR}

FLAIR Sequenzen sind aufgrund ihrer hohen Flüssigkeitssensitivität zur Detektion von Ödem und bereits demarkiertem Gewebe im Falle eines ischämischen Schlaganfalls besser geeignet als T2w Sequenzen. Sie sind daher fester und unverzichtbarer Bestandteil eines Schlaganfall MRT Protokolls. Mittels FLAIR lassen sich bereits ischämisch demarkierte Areale (=meist bereits irreversibel geschädigt) nachweisen, die noch nicht notwendigerweise in der T2w Sequenz abgrenzbar sind. So ist die FLAIR massgeblich an der Selektion der optimalen Therapie beim akuten Schlaganfall beteiligt.

\section{DWI}

Die DWI gilt als wichtigste Sequenz in der Schlaganfalldiagnostik. Das zytotoxische Ödem eines ischämischen Infarktes führt binnen Minuten zu einer nachweisbaren Einschränkung der normalen Beweglichkeit von Wassermolekülen im Hirnparenchym. Der Nachweis einer eingeschränkten Diffusion (Diffusionsrestriktion) ist mittels DWI 
äussert sensitiv und schneller als in den konventionellen MRT Sequenzen oder im nativ CT. Zu beachten ist, dass die Diffusionsbildgebung aus zwei Datensätzen besteht, die notwendigerweise immer in Zusammenschau betrachtet werden müssen: Das eigentliche DWI Bild und das dazugehörige, von der Scannersoftware automatisch generierte apparent diffusion coefficient Bild (ADC), das ein Mass für die Beweglichkeit von Wassermolekülen darstellt. Ein hohes Signal im DWI Bild (= hell) und ein korrespondierendes niedriges Signal (= dunkel) im ADC Bild entspricht einem ischämischen Infarkt. Im zeitlichen Verlauf normalisiert sich das Signal in der DWI wieder mit sinkendem Signal im DWI Bild und steigendem Signal in der ADC, so dass die Diffusionsbildgebung vor allem zum Nachweis von akuten und subakuten Infarkten verwendet wird. Ist der genaue Zeitpunkt des Symptombeginns unklar (z.B. beim nächtlichen Wake-Up Stroke) so können unter Einbezug der DWI und der FLAIR Sequenz wichtige Informationen abgeleitet werden: Ist die DWI Läsion noch nicht in der FLAIR hyperintens demarkiert, so ist von einem relativ zeitnahen Ereignis auszugehen und eine Therapie zur Revaskularisation ist als sicher und effektiv zu werten, um einen weiteren Zelluntergang zu unterbinden $(21,22)$.

\section{SWI}

In der suszeptibilitätsgewichtete Bildgebung lassen sich Blutungen mit höchster Sensitivität nachweisen. Damit ist es nicht nur möglich einen hämorrhagischen von einem ischämischen Infarkt zu differenzieren, sondern auch kleinste sekundäre hämorrhagische Einblutungen (Mikroblutungen) in ischämischen Infarkten nachzuweisen.

\section{MR-Angiographie}

Die häufigste Technik ist hierbei die TOF Angiographie, die die KM-freie Darstellung vor allem der intrakraniellen Gefässe erlaubt. Ähnlich wie bei der CT-A lassen sich so Gefässabbrüche, Stenosen oder anderweitige Gefässpathologien nachweisen. Zur Darstellung der Halsgefässe wird meist eine KM-gestützte 3D-Gradienten-Echo MRAngiographie verwendet. TOF und kontrastmittelgestützte MR-A zeigen insgesamt eine etwas geringere Sensitivität im Vergleich zur CT-A (23). Insbesondere langsamer oder turbulenter Blutfluss können zu einem Signalverlust führen, wodurch Stenosen in der MR-A überschätzt werden können (24). Bei der Frage nach Dissektion der Halsgefässe bieten sich sowohl die CT-A als auch die MR-A mit relativ ähnlichen Ergebnissen an (25). In weniger dringlichen Situationen bietet die MRT Untersuchung jedoch den Vorteil der genauen Darstellung eines möglichen Wandhämatoms und zudem mittels DWI die zeitgleiche Abklärung möglicherweise stattgefundener Infarkte. 
Weitere Vorteile der MR-A und CT-A sind die mittlerweile schnell und halbautomatisiert anzufertigenden 3-D Rekonstruktionen, mittels deren Hilfe sich schnell ein Überblick über die Gefässsituation verschafft werden kann (Abb. 4d, 5e).

\section{MR-Perfusion}

Es stehen unterschiedliche MR-Techniken zur Messung der cerebralen Perfusion zur Verfügung. Im Alltag hat sich die dynamic susceptibility contrast (DSC) Perfusion bewährt, die auf T2* ${ }^{*}$, KM-gestützten Sequenzen basiert. Das Konzept der MR-P ist ähnlich zu dem der CT-P mit analogen farbcodierten Karten mit den Parameter TTP, MTT, CBF und CBV. Auch hier soll hypoperfundiertes Hirngewebe detektiert werden um damit die optimale Patientenversorgung zu festlegen zu können. Mittels DWI kann der Infarktkern, also das irreversibel geschädigte Nervengewebe schnell und einfach definiert werden. Die zusätzlich entscheidende Frage nach einer Penumbra, also dem Vorhandensein von noch rettbarem Gewebe, kann unter Einbezug der MR-P beantwortet werden (26). Die Relation zwischen zeitlich verzögert durchblutetem Hirngewebe (definiert z.B. durch Tmax) zum irreversiblen Infarktkern ist zur Therapieentscheidung insbesondere ausserhalb der üblichen Zeitfenster relevant. Dieses Konzept wurde in neuesten Studien mehrfach belegt und untermauert den zunehmenden Wert der mechanischen Thrombektomie (8).

\section{Schlussfolgerung}

Im dynamischen Feld der Schlaganfalltherapie hat sich die fortgeschrittene Bildgebung als entscheidend bei der Diagnose und bei der Wahl der optimalen Therapie etabliert. CT ist aufgrund seiner breiten Verfügbarkeit nach wie vor die am häufigsten verwendete Modalität in der Akutsituation. MRT, soweit in der Akutsituation vorhanden, liefert ähnlich gute Ergebnisse. MRT bietet zusätzliche Optionen und ist aufgrund seiner besseren Weichteildarstellbarkeit und seiner strahlungsfreien Bildakquisition vor allem bei subakuten und späteren Infarktstadien die Methode der Wahl.

Die Neuroradiologie des Schlaganfalls ist ein sich rapide wandelndes Feld. Technische Fortschritte im Bereich der Scanner (z.B. Dual-Energy CT oder höhere Feldstärken im MRT) und Weiterentwicklungen der Bildnachbearbeitung und Bildanalyse (Stichwort künstliche Intelligenz) lassen diese Neuerungen schnell voranschreiten und auf eine künftig weitere positive Entwicklung zugunsten des Patienten hoffen.

\section{Literatur}


1. Johnson W, Onuma O, Owolabi M, Sachdev S. Stroke: a global response is needed. Bull World Health Organ. 2016;94(9):634-A.

2. Gostynski M, Engelter S, Papa S, Ajdacic-Gross V, Gutzwiller F, Lyrer P. Incidence of first-ever ischemic stroke in the Canton Basle-City, Switzerland: a population-based study 2002/2003. J Neurol. 2006;253(1):86-91.

3. Meyer K, Simmet A, Arnold M, Mattle H, Nedeltchev K. Stroke events, and case fatalities in Switzerland based on hospital statistics and cause of death statistics. Swiss Med Wkly. 2009;139(5-6):65-9.

4. Snozzi P, Blank PR, Szucs TD. Stroke in Switzerland: social determinants of treatment access and cost of illness. J Stroke Cerebrovasc Dis. 2014;23(5):926-32.

5. Pavlina AA, Radhakrishnan R, Vagal AS. Role of Imaging in Acute Ischemic Stroke. Semin Ultrasound CT MR. 2018;39(5):412-24.

6. Heldner MR, Kaesmacher J, Mordasini P, Fischer U. [Acute ischaemic stroke - Update]. Ther Umsch. 2018;75(7):411-9.

7. Ma H, Campbell BCV, Parsons MW, Churilov L, Levi CR, Hsu C, et al. Thrombolysis Guided by Perfusion Imaging up to 9 Hours after Onset of Stroke. N Engl J Med. 2019;380(19):1795-803.

8. Albers GW, Marks MP, Kemp S, Christensen S, Tsai JP, Ortega-Gutierrez S, et al. Thrombectomy for Stroke at 6 to 16 Hours with Selection by Perfusion Imaging. N Engl J Med. 2018;378(8):708-18.

9. Nogueira RG, Jadhav AP, Haussen DC, Bonafe A, Budzik RF, Bhuva P, et al. Thrombectomy 6 to 24 Hours after Stroke with a Mismatch between Deficit and Infarct. N Engl J Med. 2018;378(1):11-21.

10. Menjot de Champfleur N, Saver JL, Goyal M, Jahan R, Diener HC, Bonafe A, et al. Efficacy of Stent-Retriever Thrombectomy in Magnetic Resonance Imaging Versus Computed Tomographic Perfusion-Selected Patients in SWIFT PRIME Trial (Solitaire FR With the Intention for Thrombectomy as Primary Endovascular Treatment for Acute Ischemic Stroke). Stroke. 2017;48(6):1560-6.

11. Kim JT, Cho BH, Choi KH, Park MS, Kim BJ, Park JM, et al. Magnetic Resonance Imaging Versus Computed Tomography Angiography Based Selection for Endovascular Therapy in Patients With Acute Ischemic Stroke. Stroke. 2019;50(2):365-72.

12. Chalela JA, Kidwell CS, Nentwich LM, Luby M, Butman JA, Demchuk AM, et al. Magnetic resonance imaging and computed tomography in emergency assessment of patients with suspected acute stroke: a prospective comparison. Lancet. 2007;369(9558):293-8.

13. BAG. Strahlendosen in der Medizin 2020 [28.01.2019]. Available from: https://www.bag.admin.ch/bag/de/home/gesund-leben/umwelt-und-gesundheit/strahlungradioaktivitaet-schall/strahlenanwendungen-in-der-medizin/strahlendosen-in-dermedizin.html.

14. Winklhofer S, Andreisek G. [Imaging for back pain]. Ther Umsch. 2013;70(9):529-36.

15. Allen LM, Hasso AN, Handwerker J, Farid H. Sequence-specific MR imaging findings that are useful in dating ischemic stroke. Radiographics. 2012;32(5):1285-97; discussion 97-9. 16. Lev MH, Farkas J, Gemmete JJ, Hossain ST, Hunter GJ, Koroshetz WJ, et al. Acute stroke: improved nonenhanced CT detection--benefits of soft-copy interpretation by using variable window width and center level settings. Radiology. 1999;213(1):150-5.

17. Nakano S, Iseda T, Kawano H, Yoneyama T, Ikeda T, Wakisaka S. Correlation of early CT signs in the deep middle cerebral artery territories with angiographically confirmed site of arterial occlusion. AJNR Am J Neuroradiol. 2001;22(4):654-9.

18. Rodallec MH, Marteau V, Gerber S, Desmottes L, Zins M. Craniocervical arterial dissection: spectrum of imaging findings and differential diagnosis. Radiographics. 2008;28(6):1711-28.

19. Turc G, Bhogal P, Fischer U, Khatri P, Lobotesis K, Mazighi M, et al. European Stroke Organisation (ESO)- European Society for Minimally Invasive Neurological Therapy (ESMINT) 
guidelines on mechanical thrombectomy in acute ischemic stroke. J Neurointerv Surg. 2019;11(6):535-8.

20. Powers WJ, Rabinstein AA, Ackerson T, Adeoye OM, Bambakidis NC, Becker K, et al. 2018 Guidelines for the Early Management of Patients With Acute Ischemic Stroke: A Guideline for Healthcare Professionals From the American Heart Association/American Stroke Association. Stroke. 2018;49(3):e46-e110.

21. Thomalla G, Cheng B, Ebinger M, Hao Q, Tourdias T, Wu O, et al. DWI-FLAIR mismatch for the identification of patients with acute ischaemic stroke within $4.5 \mathrm{~h}$ of symptom onset (PRE-FLAIR): a multicentre observational study. Lancet Neurol. 2011;10(11):978-86.

22. Huisa BN, Liebeskind DS, Raman R, Hao Q, Meyer BC, Meyer DM, et al. Diffusionweighted imaging-fluid attenuated inversion recovery mismatch in nocturnal stroke patients with unknown time of onset. J Stroke Cerebrovasc Dis. 2013;22(7):972-7.

23. Bash S, Villablanca JP, Jahan R, Duckwiler G, Tillis M, Kidwell C, et al. Intracranial vascular stenosis and occlusive disease: evaluation with $C T$ angiography, MR angiography, and digital subtraction angiography. AJNR Am J Neuroradiol. 2005;26(5):1012-21.

24. Netuka D, Belsan T, Broulikova K, Mandys V, Charvat F, Malik J, et al. Detection of carotid artery stenosis using histological specimens: a comparison of $\mathrm{CT}$ angiography, magnetic resonance angiography, digital subtraction angiography and Doppler ultrasonography. Acta Neurochir (Wien). 2016;158(8):1505-14.

25. Provenzale JM, Sarikaya B. Comparison of test performance characteristics of MRI, MR angiography, and CT angiography in the diagnosis of carotid and vertebral artery dissection: a review of the medical literature. AJR Am J Roentgenol. 2009;193(4):1167-74.

26. Campbell BCV, Ma H, Ringleb PA, Parsons MW, Churilov L, Bendszus M, et al. Extending thrombolysis to 4.5-9 $\mathrm{h}$ and wake-up stroke using perfusion imaging: a systematic review and meta-analysis of individual patient data. Lancet. 2019;394(10193):139-47.

\section{Tabellen}

Tabelle 1: Vergleich der Bildgebungsmodalitäten in der Schlaganfalldiagnostik.

\begin{tabular}{|l|l|l|}
\hline & CT/CTA/CTP & MRT/MRA/MRP \\
\hline Verfügbarkeit 24/7 & Meistens & Eher selten \\
\hline Zeit-Leistungsfähigkeit & Sehr schnell & $\begin{array}{l}\text { Mit allen } \\
\text { Vorbereitungen } \\
\text { etwas langsamer }\end{array}$ \\
\hline Grössere Blutungen & $\begin{array}{l}\text { Sehr sensitiv und } \\
\text { spezifisch }\end{array}$ & $\begin{array}{l}\text { Sehr sensitiv und } \\
\text { spezifisch }\end{array}$ \\
\hline Mikroblutungen & Nicht sensitiv & $\begin{array}{l}\text { Sehr sensitiv und } \\
\text { spezifisch }\end{array}$ \\
\hline Akute Ischämiezeichen & Expertise abhängig & $\begin{array}{l}\text { Sehr eindeutig und } \\
\text { spezifisch }\end{array}$ \\
\hline $\begin{array}{l}\text { Subakute-Chronische } \\
\text { Ischämiezeichen }\end{array}$ & $\begin{array}{l}\text { Eindeutig und } \\
\text { spezifisch }\end{array}$ & $\begin{array}{l}\text { Sehr eindeutig und } \\
\text { spezifisch }\end{array}$ \\
\hline
\end{tabular}




\begin{tabular}{|l|l|l|}
\hline Gefässdarstellung & Sehr genau & Weniger detailliert \\
\hline $\begin{array}{l}\text { Darstellung von } \\
\text { Veränderungen im } \\
\text { Vertebro-basilären } \\
\text { System }\end{array}$ & Nicht sensitiv & $\begin{array}{l}\text { Sehr eindeutig und } \\
\text { spezifisch }\end{array}$ \\
\hline Patientenkooperation & Wenig abhängig & Sehr abhängig \\
\hline $\begin{array}{l}\text { Darstellbarkeit Stroke- } \\
\text { Mimiker }\end{array}$ & Nicht sensitiv & Sensitiv \\
\hline Kontraindikationen & $\begin{array}{l}\text { KM-Allergie, } \\
\text { Niereninsuffizienz }\end{array}$ & $\begin{array}{l}\text { KM-Allergie, } \\
\text { Niereninsuffizienz, } \\
\text { Metall-Implantate }\end{array}$ \\
\hline
\end{tabular}

Tabelle 2: Altersstadien (ischämischer) Schlaganfall

\begin{tabular}{|l|l|}
\hline $\begin{array}{l}\text { Altersstadium (ischämischer) } \\
\text { Infarkt }\end{array}$ & Zeit \\
\hline Früh hyperakut & 0 bis 6 Stunden \\
\hline Spät hyperakut & 6 bis 24 Stunden \\
\hline Akut & 24 Stunden bis 1 Woche \\
\hline Subakut & 1 bis 3 Wochen \\
\hline Chronisch & Mehr als 3 Wochen \\
\hline
\end{tabular}

Tabelle 3: Parameter der Perfusionsbildgebung (CT und MRT)

\begin{tabular}{|l|l|l|}
\hline Parameter & Definition & Einheit \\
\hline CBF & $\begin{array}{l}\text { Menge an Blut (ml), die pro Masse Gewebe } \\
\text { (g) pro Zeit (min) fliesst. }\end{array}$ & $\mathrm{ml} / 100 \mathrm{~g} / \mathrm{min}$ \\
\hline CBV & Menge an Blut (ml) pro Masse Gewebe $(\mathrm{g})$ & $\mathrm{ml} / 100 \mathrm{~g}$ \\
\hline TPP & $\begin{array}{l}\text { Benötigte Zeit bis zu der die KM- } \\
\text { Konzentration ihr Maximum erreicht hat }\end{array}$ & $\mathrm{s}$ \\
\hline MTT & $\begin{array}{l}\text { Benötigte Zeit für KM um aus einer Arterie in } \\
\text { durch das Gewebe in ein venöses Gefäss } \\
\text { überzutreten. }\end{array}$ & $\mathrm{s}$ \\
\hline Tmax & $\begin{array}{l}\text { Zeitpunkt des Maximums der so genannten } \\
\text { Restfunktion }\end{array}$ & $\mathrm{s}$ \\
\hline
\end{tabular}

CBF $=$ Cerebral Blood Flow, CBV $=$ Cerebral Blood Volume, TTP $=$ Time-to-peak, MTT = Mean Transit Time, Tmax $=$ Time-to-maximum, $\mathrm{KM}=$ Kontrastmittel

\section{Abbildungen}

Abbildungen separat beigefügt. 
Abb. 1: Beispiele intrakranieller Blutungen im axialen nativ CT in Abhängigkeit ihrer Lokalisation. Intra-axiale Blutungen: a) Hypertensiv bedingte

Stammganglienblutung mit zusätzlichem Ventrikeleinbruch, b) Lobärblutung (bei cerebraler Amyloidangiopathie), c) Kleinhirnblutung (zugrunde liegende Metastase). Extra-axiale Blutungen: d) Epiduralhämatom (EDH), e) Subduralhämatom (SDH), f) Subarachnoidalblutung (SAB).
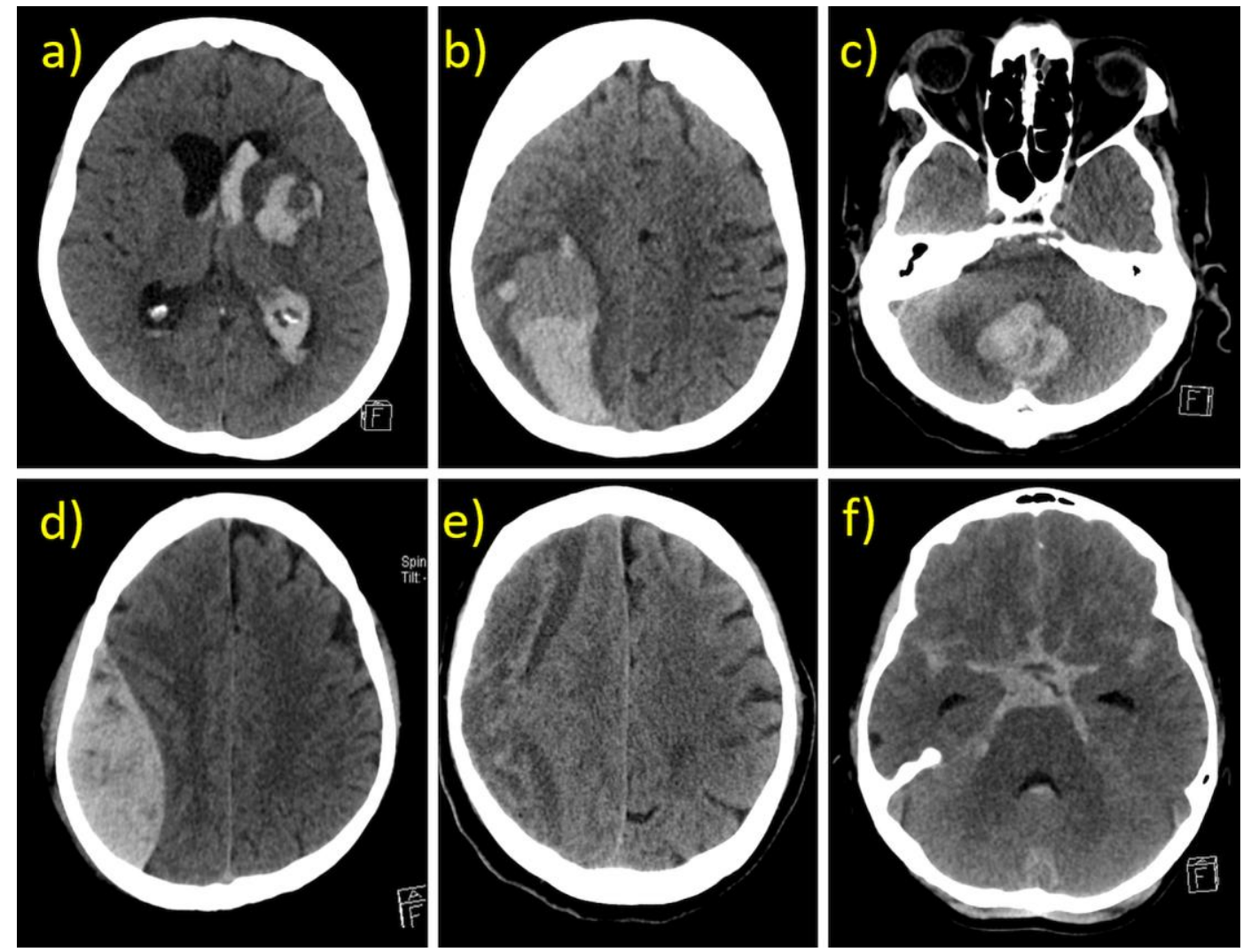

Abb. 2: Infarktfrühzeichen im axialen nativ CT (a-d) und Korrelation zum MRT (e, f). a) hyperdenses Arterien Zeichen (Pfeil) bei Thrombus in der A. cerebri media rechts.

b) Verminderte Differenzierbarkeit der Grauen und Weissen Substanz mit einhergehender flau-hypodenser Parenchymschwellung sowie verminderte Abgrenzbarkeit der Basalganglien (Pfeile); c) selber Patient 6 Monate später nun Infarkt im chronischen Stadium mit etwas grösserer Ausdehnung als im initialen Bild. d) Verstrichenes Mark-Rinden Band (Pfeile) mit Korrelat in der MRT Untersuchung, e) 
DWI, f) ADC.
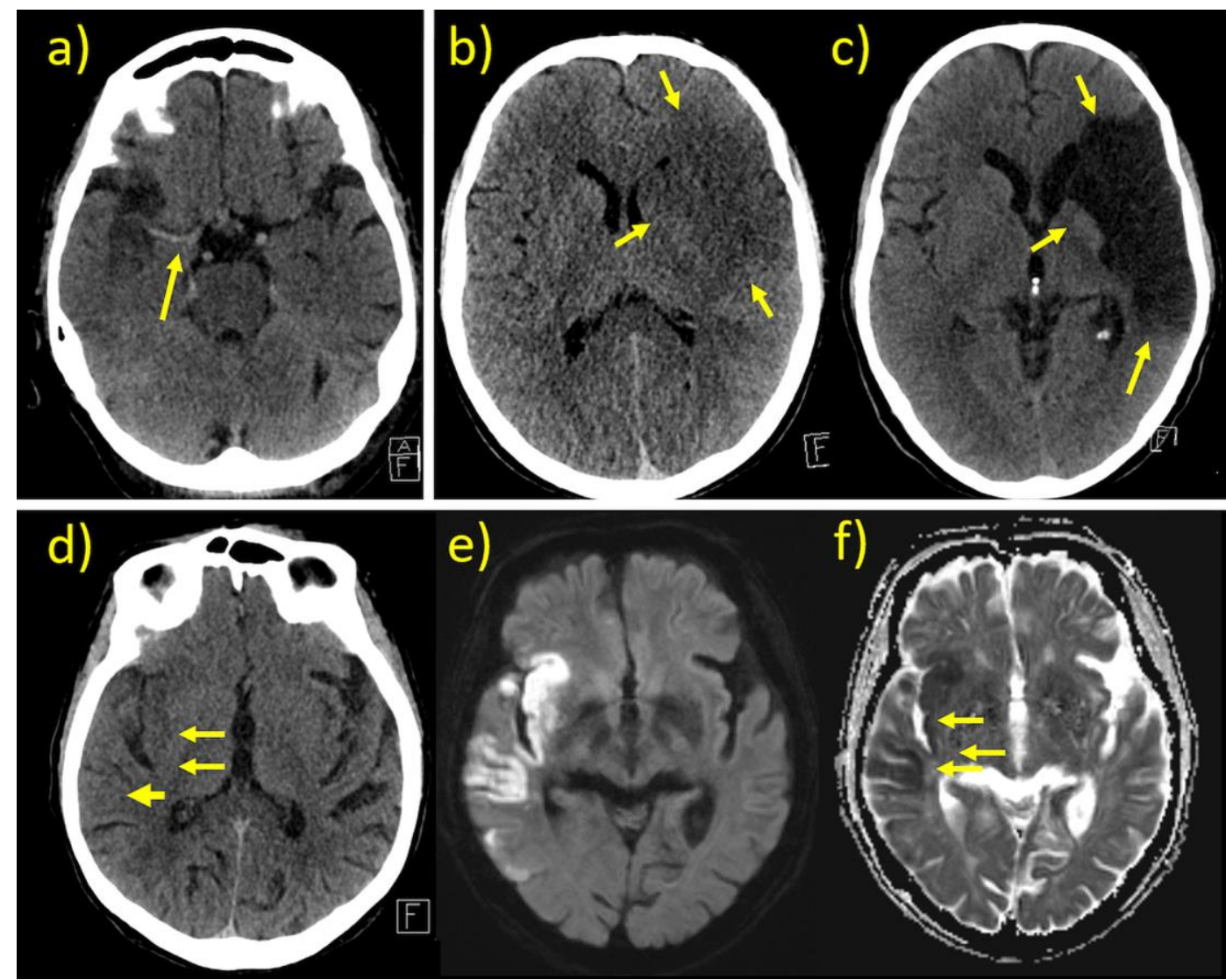

Abb. 3: Das Konzept der penumbralen Perfusionsbildgebung. Das rote Areal kennzeichnet den Infarktkern, das grüne Areal das minderperfundierte Gebiet (Penumbra, rettbares Gewebe). Der Unterschied zwischen Infarktkern und Penumbra (= Mismatch) beeinflusst die Indikation für die Revaskularisation. a) grosses Mismatch, guter, b) kleines oder fehlendes Mismatch, ungünstiger Kandidat für Revaskularisation. Bei ausbleibender Revaskularisation besteht die Gefahr der Volumenzunahme des Infarktkernes.

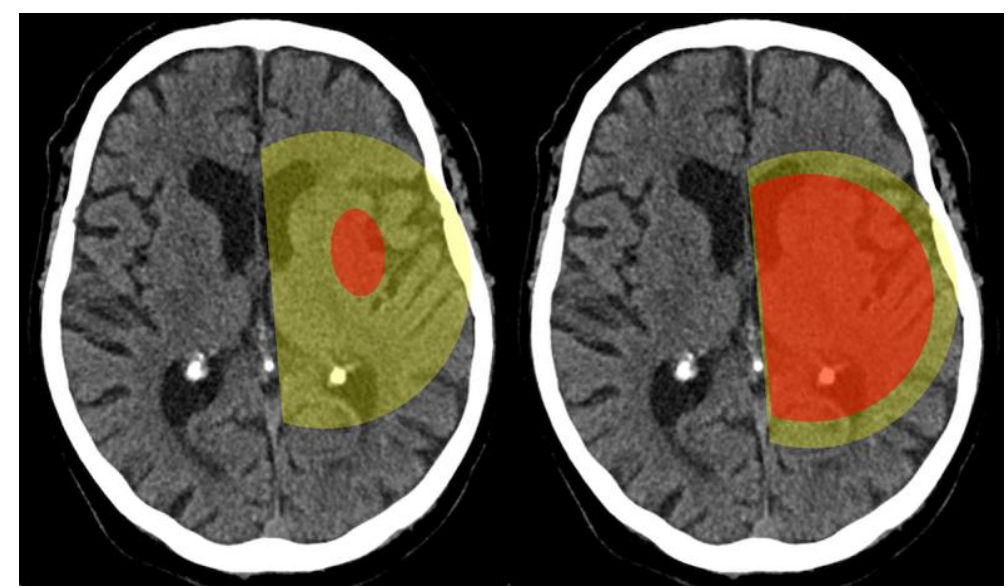


Abb. 4: 79-jähriger Patient mit Hemiparese rechts seit zwei Stunden. a) Nativ CT ohne sicheren Nachweis von Infarktfrühzeichen. In der CT-Perfusion Nachweis einer deutlich verzögerten Durchblutung im Mediaterritorium links in der Tmax b) jedoch ohne wesentlich vermindertes Blutvolumen in der CBV c), einem grossen Mismatch entsprechend. d) Verschluss der distalen A. cerebri media links (3D Rekonstruktion der CT-Angiographie). e) Aufgrund dieser Konstellation Entscheid zur umgehenden neurointerventionellen mechanischen Thrombektomie mit erfolgreicher kompletter Revaskularisation des Gefässes in der DSA. f) Die Follow up MRT Untersuchung einen Tag nach Ereignis zeigt eine unauffällige Diffusionsbildgebung ohne Nachweis von ischämischen Läsionen.

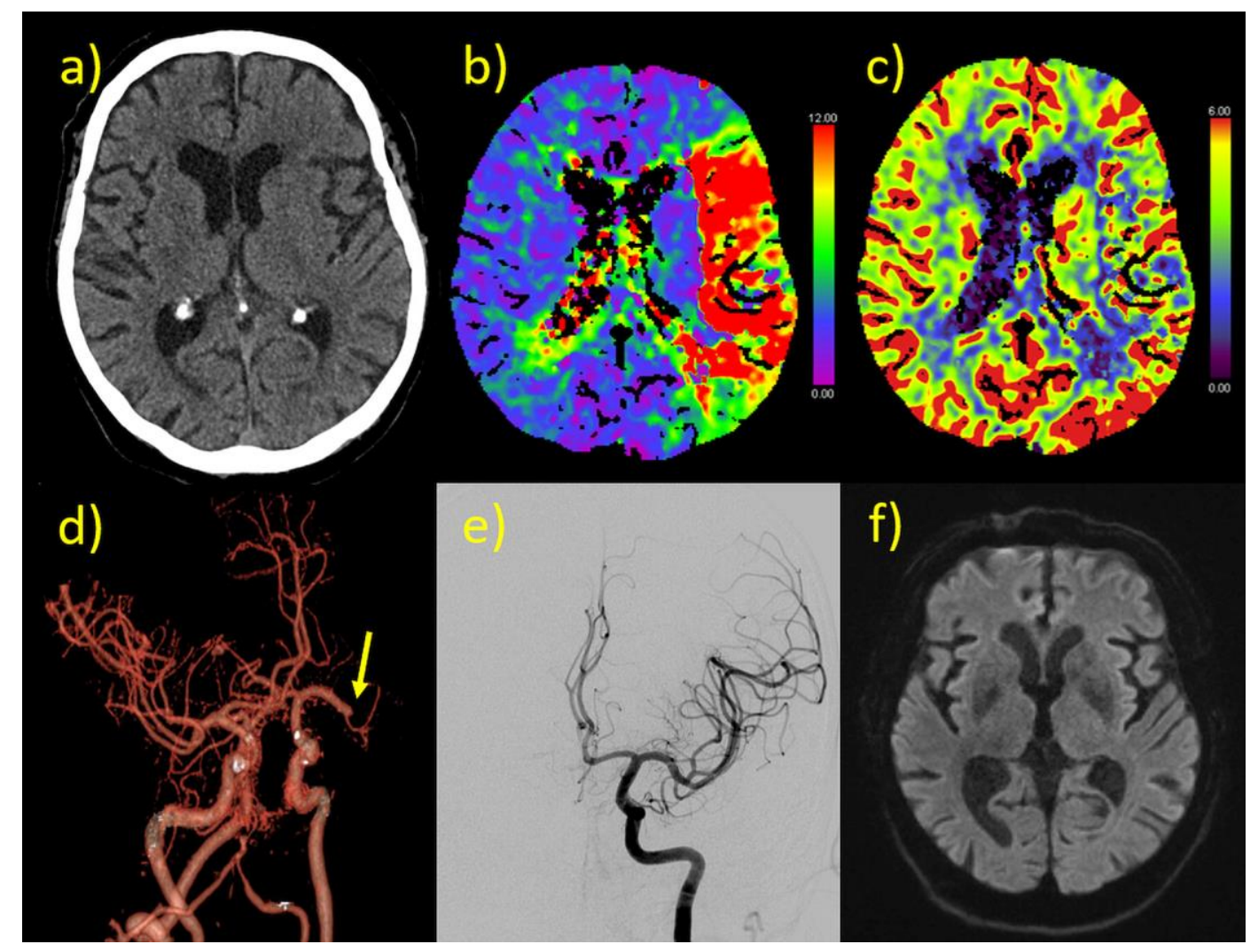

Abb. 5: 59-jähriger Patient mit neu aufgetretener, globaler Aphasie. MRT im Rahmen der initialen Abklärung: Flächige Diffusionsrestriktion in der DWI Bildgebung; DWI a), ADC b). Das ischämisch infarzierte Areal lässt sich bereits hyperintens in der T2w c) und FLAIR d) nachweisen. Ursächlich für die Ischämie zeigt sich eine Dissektion der A. carotis interna links mit langstreckigem Gefässverschluss in der Halsangiographie 
(Pfeil in e). Zusätzlich Verschluss des M2 Segmentes der A. cerebri media in der TOFAngiographie f). An dieser Lokalisation Nachweis eines längeren Thrombus in der SWI g). Deutlich verzögerte Perfusion in der TTP h) ohne Nachweis eines Mismatch im Vergleich zum infarzierten Gewebe in der DWI a), b).

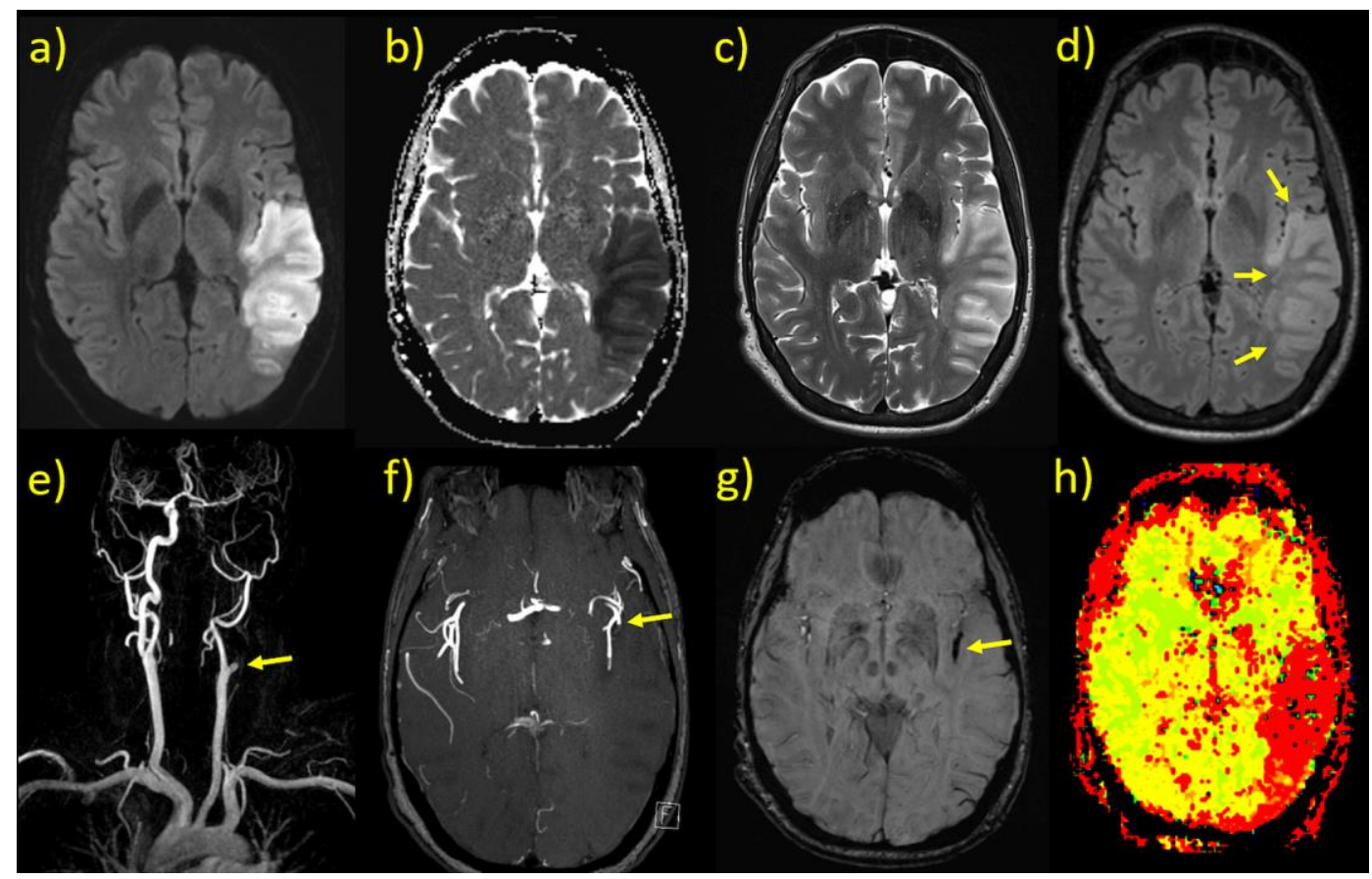

